Maria Alice SeGhessi AlbinO ${ }^{1}$

ANA SILVIA MOCCELLIN²

BeATRIZ da SILVA FIRMENTO ${ }^{3}$

PATRICIA DRIUSSO 4

Artigo Original

Palavras-chave

Gravidez Índice de massa corporal

Pé

Marcha

Biomecânica

Keywords

Pregnancy

Body mass index

Foot

Gait

Biomechanics

\title{
Modificações da força de propulsão da marcha durante a gravidez: efeito das alterações nas dimensões dos pés
}

\author{
Gait force propulsion modifications during \\ pregnancy: effects of changes in feet's dimensions
}

\section{Resumo}

OBJETIVOS: analisar a força de propulsão na marcha e relacionar com as alterações nas dimensões dos pés e a influência sobre a qualidade de vida da gestante. MÉTODOS: dois grupos, um Controle (C), com 20 mulheres voluntárias não grávidas, e outro com 13 gestantes voluntárias que foram avaliadas nos três trimestres gestacionais (Gpri, Gseg, Gter). Os grupos foram submetidos a uma avaliação inicial; a uma avaliação da força de propulsão da marcha por meio da plataforma de força (Bertec); avaliação do comprimento e largura dos pés feita por técnicas de metragem; avaliação da perimetria realizada pelo método "figura em oito"; e avaliação da qualidade de vida obtida pelo Questionário World Health Organization Quality of Life Instrument Bref (Whogol-bref). Foi utilizado o teste de Mann-Whitney para avaliar as diferenças entre o grupo C e Gpri; entre Gpri, Gseg e Gter utilizou-se o teste de Friedman e nos casos significativos aplicou-se o teste de Wilcoxon. Foi considerado um nível de significância de 5\%. RESULTADOS: aumento da massa corporal $(10,5 \mathrm{~kg})$ e do edema dos tornozelos $(2,4 \mathrm{~cm})$ ao longo da gestação; observa-se diminuição da força de propulsão da marcha (10\% da massa corporal) e maior oscilação mediolateral (10\% da massa corporal) comparadas ao Grupo Controle; queda na qualidade de vida da gestante, principalmente no domínio físico. CONCLUSÃO: ocorrem alterações na marcha durante a gestação, levando a um risco maior de quedas e desconfortos musculoesqueléticos que pode influenciar na qualidade de vida da gestante.

\section{Abstract}

PURPOSE: to analyze the gait propulsion force and relate it to changes in the dimensions of the feet and to the influence on the quality of life of pregnant women. METHODS: two groups were studied, a control (C) one consisting of 20 non-pregnant women and a group of 13 pregnant women investigated during the three gestational trimesters (Gfirst, Gsecond, Gthird). The groups were subjected to an initial assessment; evaluation of gait propulsion force using the force platform (Bertec); measurement of foot length and width; assessment of perimetry by the figure eight method; and assessment of quality of life using the World Health Organization Quality of Life Instrument Bref (Whogol-bref). The MannWhitney test was used to evaluate differences between group $C$ and Gfirst, the Friedman test was used to determine differences between Gfirst, Gsecond and Gthird, and the Wilcoxon test was applied to significant cases. The level of significance was set at $5 \%$. RESULTS: There was an increase in body mass $(10.5 \mathrm{~kg})$ and ankle edema $(2.4 \mathrm{~cm})$ during pregnancy. There was a decrease of gait propulsion force (10\% of body mass) and an increase of mediolateral sway (10\% of body mass) compared to Control Group. There was a reduced quality of life among pregnnat women, especially in the physical domain. CONCLUSIONS: Gait disorders occur during pregnancy, which can increase the risk of falls and musculoskeletal discomfort, which may affect the quality of life of pregnant women.

Correspondência:

Mario Alice Seghessi Albino Universidade Federal de São Carlos - UFSCar Via Washington Luis, $\mathrm{km} 235, \mathrm{CP} 676$ (EP: 13565-905 - São Carlos (SP), Brasil

Recebido 21/06/2011
Laboratório de Avaliação Biomecânica, Aprendizagem e Treinamento (LABAT), junto ao Núcleo Multidisciplinar para Análise do Movimento (NAM), dos Departamentos de Fisioterapia e Educação Física da Universidade Federal de São Carlos - UFSCar - São Carlos (SP), Brasil.

' Fisioterapeuta da Universidade Federal de São Carlos - UFSCar - São Carlos (SP), Brasil.

${ }^{2}$ Aluna de Pós-graduação (Doutorado) em Fisioterapia pelo Programa de Pós-Graduação da Universidade Federal de São Carlos UFSCar - São Carlos (SP), Brasil.

${ }^{3}$ Acadêmico em Fisioterapia pela Universidade Federal de São Carlos - UFSCar - São Carlos (SP), Brasil.

${ }_{4}^{4}$ Professor Doutor do Departamento de Fisioterapia da Universidade Federal de São Carlos - UFSCar - São Carlos (SP), Brasil. 


\section{Introdução}

O sistema musculoesquelético é afetado durante o período gestacional devido às alterações hormonais, bioquímicas e anatômicas ${ }^{1,2}$, podendo gerar, entre outros fatores, adaptações na marcha. Além disso, podem ocorrer alterações nas dimensões dos pés da gestante ${ }^{3}$ como: aumento do comprimento, largura e volume, e a redução da altura do arco plantar ${ }^{4}$. Os maiores responsáveis por essas alterações são o acúmulo de líquido, a frouxidão ligamentar e o ganho de massa corporal ${ }^{5}$.

Ao longo da gestação, $80 \%$ das mulheres acumulam líquidos e são retidos, aproximadamente, seis litros e meio de fluidos. Outro mecanismo de predisposição ao edema durante a gravidez é o gravitacional, consequente da pressão exercida sobre os capilares dos membros inferiores e pela compressão da veia cava inferior pelo útero gravídico ${ }^{6}$.

Já a ocorrência de frouxidão ligamentar está relacionada à intensa ação dos hormônios progesterona, estrógeno, cortisol e relaxina, que é o principal fator do aumento de mobilidade articular ${ }^{7}$. A relaxina é um polipeptídeo produzido pelo corpo lúteo e atua diretamente sobre o tecido conectivo, estimulando a expressão da colagenase e a síntese de colágeno. No período gestacional, sua atuação caracteriza-se pelo aumento da mobilidade articular, principalmente na sínfise púbica ${ }^{8}$.

Quanto ao ganho de massa corporal, a média ideal durante a gestação é de 11 quilos $^{9}$. Porém, esse valor varia muito e apenas de 30 a $40 \%$ das gestantes ganham a quantidade recomendada de massa durante a gravidez. Assim, quanto maior o ganho de massa corporal, maior acúmulo de gordura após o metabolismo, podendo levar a alterações morfológicas persistentes após o parto ${ }^{10}$. Por exemplo, um ganho de massa corporal de $20 \%$ durante a gestação gera sobrecarga nas articulações que pode corresponder a $100 \%$ do peso total ${ }^{11}$.

Como cerca de metade da massa corporal adquirida na gestação concentra-se na área abdominal, anterior à linha de gravidade, o centro de gravidade (CG) é deslocado anteriormente. Somado a esse fator, o ganho de massa e as modificações nas dimensões dos pés podem levar a desvios da força de reação do solo (FRS) e da força de propulsão, presentes na marcha ${ }^{12}$, resultando em perda do equilíbrio e, consequentemente, em traumas, como quedas ${ }^{13}$.

Em um estudo, observou-se o aumento da base de suporte na marcha das grávidas durante a caminhada e foi atribuído a isso um mecanismo compensatório para melhorar a estabilidade locomotora ${ }^{14}$. Porém, outro estudo evidenciou que o padrão de marcha em gestantes permaneceu relativamente inalterado, mesmo com o acréscimo de massa corporal, o que indica a ocorrência de uma possível sobrecarga sobre os músculos abdutores e extensores da pelve e sobre os músculos responsáveis pela flexão plantar do tornozelo ${ }^{15}$.

As contradições entre os estudos citados, além da escassez de pesquisas caracterizando as alterações ocorridas nos pés e na marcha das gestantes, evidenciam a necessidade de mais trabalhos para averiguar a ocorrência de adaptações na marcha durante a gestação, e verificar se estas estão relacionadas com as possíveis mudanças nas dimensões dos pés ao longo da gravidez. Dessa forma, o estudo tem como objetivo verificar a ocorrência de alterações nas dimensões dos pés das gestantes e relacioná-las com a diminuição da força de propulsão na fase de apoio da marcha nos diferentes trimestres gestacionais.

\section{Métodos}

O estudo foi aprovado pelo Comitê de Ética em Pesquisa em Seres Humanos da Universidade Federal de São Carlos - UFSCar, conforme determina a resolução 196/96 do Conselho Nacional de Saúde, sob o parecer $\mathrm{n}^{\circ} .280 / 2009$.

\section{Sujeitos}

Todas as voluntárias assinaram o Termo de Consentimento Livre e Esclarecido. O critério para a seleção determinou que tanto as voluntárias gestantes quanto as não gestantes apresentassem idade entre 18 e 40 anos, fossem saudáveis, não fumantes, não etilistas e nem apresentassem cirurgias na coluna vertebral, pelve, quadril ou joelho e nenhuma condição prévia à avaliação que pudesse afetar a estabilidade postural (disfunções musculoesqueléticas ou neurológicas). Além disso, as gestantes deveriam apresentar gestação de baixo risco e de feto único, sem intercorrências do ciclo gravídico. Treze gestantes concluíram o estudo. Para estabelecimento de um controle basal foram analisadas 20 mulheres não gestantes. A Tabela 1 apresenta as características antropométricas dos grupos Controle e Gestante.

Tabela 1. Características antropométricas das participantes do Grupo Controle e Grupo Gestante

\begin{tabular}{|c|c|c|c|c|c|c|c|c|}
\hline \multirow{2}{*}{ Características } & \multicolumn{2}{|c|}{ Controle } & \multicolumn{2}{|c|}{$1^{\circ}$ trimestre } & \multicolumn{2}{|c|}{$2^{\circ}$ trimestre } & \multicolumn{2}{|c|}{$3^{\circ}$ trimestre } \\
\hline & Média & DP & Média & DP & Média & DP & Média & DP \\
\hline Semana gestacional & - & - & 13,1 & 2,1 & 23,2 & 1,6 & 33,4 & 1,2 \\
\hline Idade (anos) & - & 4,0 & 29,1 & 5,6 & 29,2 & 5,8 & 29,5 & 5,8 \\
\hline Altura (m) & 1,6 & 0,1 & 1,6 & 0,1 & 1,6 & 0,1 & 1,6 & 0,09 \\
\hline Peso (kg) & 59,2 & 10,7 & 66,2 & 13,3 & 71,3 & 13,5 & 76,7 & 14,9 \\
\hline IMC $\left(\mathrm{kg} / \mathrm{m}^{2}\right)$ & 22,2 & 3,6 & 24,6 & 4,4 & 26,5 & 4,3 & 28,5 & 4,7 \\
\hline
\end{tabular}

DP: desvio padrão. 


\section{Procedimentos experimentais}

Todas as mulheres foram submetidas a uma avaliação inicial por meio de entrevista individual, utilizando-se um questionário para registro dos dados pessoais, hábitos de vida, antecedentes pessoais, uso de medicamentos, história ginecológica e obstétrica.

As voluntárias do Grupo Controle (C) foram avaliadas apenas uma vez, e comparadas ao primeiro trimestre gestacional das gestantes. As gestantes foram avaliadas em três momentos distintos, entre a $12^{\mathrm{a}}-14^{\mathrm{a}}$ (Gpri), $22^{\mathrm{a}}-24^{\mathrm{a}}$ (Gseg) e 32 $-34^{\mathrm{a}}$ (Gter) semanas gestacionais, de acordo com a data da última menstruação ${ }^{16}$ e/ou do primeiro ultrassom realizado durante a gestação ${ }^{17}$.

\section{Avaliação das dimensões dos pés}

A metragem dos pés foi realizada com a voluntária sentada com os joelhos em $90^{\circ}$ de flexão e os pés posicionados sobre um papel quadriculado de $0,5 \times 0,5 \mathrm{~cm}$. Para mensurar o comprimento foi colocada uma régua no ponto mais saliente do hálux e feita uma marca no papel quadriculado. No calcanhar foi feito o mesmo procedimento e, assim, traçadas retas paralelas a partir das marcas correspondentes ao hálux e ao calcanhar. A largura foi mensurada pelo mesmo método, colocando-se a régua na área correspondente à cabeça do primeiro metatarso e à cabeça do quinto metatarso, traçando-se retas paralelas a partir das respectivas áreas, de forma que a largura foi medida no plano transversal ${ }^{5}$.

\section{Avaliação da perimetria}

A mensuração da perimetria do tornozelo nos pés foi feita a partir da técnica "figura em oito", com a voluntária em decúbito dorsal sobre a maca, joelhos em extensão completa e tornozelo em posição neutra. O ponto zero da fita métrica foi o ponto médio entre a projeção articular do tendão do músculo tibial anterior e o maléolo lateral. A partir desse ponto, a fita tangenciou a borda anterior do maléolo lateral em direção à planta do pé, seguindo para o arco longitudinal medial, onde passou por cima do osso navicular, na direção do maléolo lateral; em seguida, a fita passou sobre o tendão calcâneo e sobre o maléolo medial até encontrar o ponto zero da fita métrica ${ }^{18}$.

\section{Avaliação da força de propulsão}

$A$ avaliação da força de propulsão da marcha foi realizada na plataforma de força Bertec. A cada avaliação realizada as plataformas de força eram calibradas nas três direções (horizontal anteroposterior e mediolateral, e vertical).

As voluntárias eram instruídas a andar normalmente sobre a plataforma, utilizando um mesmo calçado confortável e particular para todas as avaliações, e fixar o olhar em um ponto no espaço posicionado na altura dos olhos. Caminharam durante 20 minutos, ou pelo tempo necessário para obtenção de cinco tentativas de marcha válidas para análise.

Com o intuito de normalizar os valores obtidos na plataforma, os resultados foram divididos pelo peso corporal do indivíduo obtido em cada avaliação, sendo fornecidos em quilograma-força. Outra medida de normalização refere-se à duração temporal da FRS, necessária para que haja comparação entre diferentes repetições e diferentes indivíduos. Dessa forma, o início dos dados da FRS corresponde a $0 \%$ e o fim a $100 \%$ e com o auxílio do recurso matemático de interpolação, foram gerados determinados números de pontos entre 0 a $100 \%$ para as diferentes repetições.

Para cada tentativa foi gerado um arquivo de texto (.txt) com dados dos três componentes de força e momentos de força. A frequência de aquisição do sinal do COP foi de $100 \mathrm{~Hz}$. Todas as voluntárias tinham o membro inferior direito como dominante.

Os parâmetros que descrevem os componentes da força de propulsão da marcha foram processados no programa MatLab Math Works versão 7.0.1.24704 (R14). Os dados foram filtrados por meio do filtro Butterworth, de primeira ordem, reduzindo a passagem de frequências acima de $5 \mathrm{~Hz}$, de forma que os dados fossem captados a partir do instante do primeiro contato do pé com a plataforma. As variáveis analisadas foram componente vertical $(\mathrm{Fz})$, contendo o valor do apoio no calcanhar ou desaceleração (Pico 1), valor do apoio nos metatarsos ou impulsão (Pico 2), valor da descarga de peso no médio-pé (Vale) e tempos do Pico 1, Pico 2 e vale. O componente horizontal foi analisado nas direções anteroposterior, a partir do valor máximo de impulsão (Max Fy) e mediolateral, contendo a diferença entre a oscilação medial e lateral (Max-min Fx). Foram utilizadas para análise estatística as médias dos valores entre as cinco tentativas.

\section{Avaliação da qualidade de vida}

A avaliação da qualidade de vida foi feita pelo Whoqol-bref, uma versão resumida do questionário World Health Organization Quality of Life Instrument 100 (Whoqol-100), desenvolvido pelo Grupo de Qualidade de Vida da Organização Mundial da Saúde. A versão nacional do Whoqol-bref foi validada e traduzida pelo Grupo de Estudos em Qualidade de Vida da Organização Mundial da Saúde do Brasil ${ }^{19}$.

O Whoqol-bref é composto por 26 questões, sendo duas questões gerais de qualidade de vida e as outras representam cada uma das 24 facetas que compõem o instrumento original, englobando os domínios físico e psicológico, relações sociais e meio ambiente ${ }^{20}$. 
O questionário apresentou um bom desempenho psicométrico permitindo, assim, ampla utilização e praticidade. Além disso, mostrou-se com características satisfatórias de consistência interna, validades discriminante, concorrente, de critério e fidedignidade teste-reteste ${ }^{19}$.

\section{Análise estatística}

Os dados foram tabulados no Excel e analisados estatisticamente no programa Statistica e por meio de técnicas descritivas (tabelas e gráficos). Os dados foram analisados por meio de testes não paramétricos, após a verificação de que algumas variáveis não seguiam uma distribuição normal, por meio do teste de Shapiro-Wilk. A comparação entre os três trimestres (Gpri, Gseg e Gter) foi realizada pelo teste de Friedman e, nos casos significantivos, utilizou-se o teste de Wilcoxon para discriminar a diferença. Para a comparação entre as gestantes do primeiro trimestre (Gpri) e não gestantes $(\mathrm{C})$ utilizou-se o teste de Mann-Whitney. Foi adotado um nível de significância de $5 \%(\mathrm{p} \leq 0,05)$.

\section{Resultados}

A Tabela 2 apresenta as características das alterações nas dimensões do membro dominante (D) e não dominante (ND) entre os trimestres gestacionais, encontrando-se diferença significativa na perimetria do membro ND entre o primeiro e terceiro trimestres gestacionais.

A Tabela 3 apresenta os resultados para a avaliação do controle postural dinâmico para o Grupo Controle e primeiro trimestre gestacional nos membros dominante e não dominante, destaca-se que só ocorreram diferenças significativas, em algumas variáveis, na comparação do Grupo Controle com o Gpri. Estão apresentadas as variáveis analisadas, relacionadas à Força de Reação do Solo (FRS): tempo e valor do ponto máximo de pressão no calcanhar no componente vertical da FRS (P1), do ponto máximo de pressão nos metatarsos no componente vertical da FRS (P2); máximo valor encontrado na direção anteroposterior do componente horizontal (Max Fy) e a diferença entre os valores máximo e mínimo na direção mediolateral do componente horizontal (max-min Fx). Os dados mostram que a partir do primeiro trimestre, a gestante demora mais para realizar o apoio total do calcanhar quando comparado ao Grupo Controle, resultando em uma diferença significativa para os membros dominantes e membros não dominantes. Esta característica permaneceu durante toda a gestação.

Com relação ao Questionário de Qualidade de Vida Whoqol-bref, ocorreu uma diferença significativa $(\mathrm{p}=0,016)$ entre o Grupo Controle e o primeiro trimestre gestacional, no domínio físico. Este domínio foi o mais afetado, diminuindo ainda mais a partir do terceiro trimestre de gestação, seguido pelos domínios psicológico, comportamental e social.
Tabela 2. Características das dimensões dos pés nos trimestres gestacionais

\begin{tabular}{|c|c|c|c|c|c|c|c|}
\hline \multirow[t]{2}{*}{ Características(cm) } & \multicolumn{2}{|c|}{$1^{\circ}$ trimestre } & \multicolumn{2}{|c|}{$2^{\circ}$ trimestre } & \multicolumn{2}{|c|}{$3^{\circ}$ trimestre } & \multirow[t]{2}{*}{ Valor $\mathrm{p}$} \\
\hline & Média & DP & Média & DP & Média & DP & \\
\hline Comprimento D & 23,8 & 1,2 & 23,8 & 1,3 & 23,9 & 1,3 & 0,81 \\
\hline Comprimento ND & 23,6 & 1,1 & 23,7 & 1,3 & 23,8 & 1,3 & 0,9 \\
\hline Largura D & 8,9 & 0,6 & 9,1 & 0,6 & 9,3 & 0,6 & 0,3 \\
\hline Largura ND & 9,1 & 0,8 & 9,1 & 0,6 & 9,3 & 0,6 & 0,6 \\
\hline Perimetria D & 49,4 & 2,5 & 50,3 & 2,7 & 51,7 & 3,5 & 0,07 \\
\hline Perimetria ND & 49,3 & 2,4 & 50,0 & 2,6 & 51,7 & 3,3 & 0,04 \\
\hline
\end{tabular}

D: membro dominante; ND: membro não dominante

Tabela 3. Resultados das variáveis relacionadas à Força de Reação do Solo (FRS) para os membros dominante e não dominante, avaliadas no Grupo Controle e primeiro trimestre gestacional

\begin{tabular}{|c|c|c|c|c|c|c|}
\hline \multirow{2}{*}{ W } & \multirow{2}{*}{ Variáveis } & \multicolumn{2}{|c|}{ Controle } & \multicolumn{2}{|c|}{$1^{\circ}$ trimestre } & \multirow{2}{*}{ Valor $p$} \\
\hline & & Média & DP & Média & DP & \\
\hline \multirow{7}{*}{ 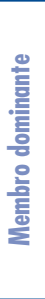 } & Tempo Pl & 0,20 & 0,02 & 0,25 & 0,05 & 0,001 \\
\hline & Tempo P2 & 0,55 & 0,04 & 0,61 & 0,06 & 0,002 \\
\hline & Tempo máximo Fy & 0,61 & 0,05 & 0,69 & 0,06 & 0,001 \\
\hline & Valor Pl & 1,08 & 0,06 & 1,05 & 0,06 & 0,196 \\
\hline & Valor P2 & 1,08 & 0,04 & 1,06 & 0,05 & 0,035 \\
\hline & Valor máximo Fy & 0,19 & 0,03 & 0,15 & 0,04 & 0,005 \\
\hline & Valor máximo-mínimo Fx & 0,34 & 0,13 & 0,23 & 0,08 & 0,004 \\
\hline \multirow{7}{*}{ 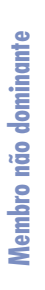 } & Tempo P1 & 0,20 & 0,03 & 0,25 & 0,05 & 0,001 \\
\hline & Tempo P2 & 0,55 & 0,04 & 0,61 & 0,05 & 0,001 \\
\hline & Tempo máximo Fy & 0,62 & 0,04 & 0,70 & 0,06 & 0,000 \\
\hline & Valor P1 & 1,10 & 0,06 & 1,05 & 0,04 & 0,008 \\
\hline & Valor P2 & 1,11 & 0,04 & 1,05 & 0,04 & 0,004 \\
\hline & Valor máximo Fy & 0,18 & 0,03 & 0,14 & 0,05 & 0,002 \\
\hline & Valor máximo-mínimo Fx & 0,21 & 0,09 & 0,19 & 0,08 & 0,577 \\
\hline
\end{tabular}

P1: ponto máximo de pressão no calcanhar no componente vertical da Força de Reação do Solo; P2: ponto máximo de pressão nos metatarsos na componente vertical da Força de Reação do Solo; Fy: força da componente horizontal na direção anteroposterior da Força de Reação do Solo; Fx: força da componente horizontal na direção mediolateral. Valores expressos em porcentagem do peso corporal.

\section{Discussão}

Os resultados apresentados demonstram que ocorreu ganho de massa corporal durante a gestação, assim como ocorreu aumento na perimetria em ambos os tornozelos, sugerindo uma forte correlação entre estas duas características, pois o acúmulo de líquido decorrente do feto, placenta, útero, mamas, líquido amniótico, extravasamento de líquido extracelular e aumento do volume do sangue, em conjunto com o crescimento fetal, são os responsáveis pelo ganho de massa corporal e também pela ocorrência do edema, que levou ao aumento da perimetria dos tornozelos ${ }^{2,5}$.

No presente estudo, foi observado que o aumento da massa corporal pode interferir nas características da marcha das gestantes, aumentando o tempo de apoio do calcanhar, o que também foi evidenciado por Foti et al. ${ }^{15}$, e diminuindo a força de propulsão da marcha, da 
componente vertical (Pico 2), quando comparado com o Grupo Controle.

Acredita-se que o maior tempo de apoio durante a fase de resposta à carga seja decorrente da diminuição na velocidade da marcha. Um estudo sugeriu que com o aumento da massa corporal por volta de $12 \mathrm{~kg}$, gera-se um limiar fisiológico que diminui a velocidade da marcha ${ }^{21}$. Outra possibilidade é que o tempo de apoio aumentado seja uma adaptação gestacional para absorver melhor o impacto.

A força de propulsão da marcha está presente na fase de apoio terminal, que corresponde ao intervalo de 30-50\% do Ciclo da Marcha (CM) que é composto por oito fases. Em condições normais esta fase começa com a elevação dos calcanhares e apoio dos metatarsos, antecedendo a fase de pré-balanço. O tornozelo encontra-se em flexão plantar de $20^{\circ}$, e toda tensão muscular está concentrada no músculo tríceps sural, que sustenta cerca de $20 \%$ a mais do peso corporal para realizar a impulsão do membro, ou seja, desencadear a Força de Propulsão ${ }^{22}$.

Uma possível explicação para a diminuição da força de propulsão durante a gestação é que, com o aumento do peso corporal, ocorre uma sobrecarga na ação muscular do tríceps sural, fazendo com que sua tensão não seja tão efetiva. Associado a esse quadro, o edema e o aumento na largura dos pés impede que a gestante faça um grau de flexão plantar adequado para realizar uma contração excêntrica e gerar um torque no músculo tríceps sural suficiente para originar a força de propulsão. Porém, um valor normal para o componente horizontal de força na direção anteroposterior da FRS (Fy) seria de $23 \%$ do peso corporal $^{22}$. Neste estudo, os valores de Fy estão em torno de $15 \%$ do peso corporal, o que significa uma diminuição, podendo ser outra explicação para a redução da força de propulsão da marcha.

Outro resultado observado com relação à força de propulsão é que, no terceiro trimestre gestacional, no membro dominante, ocorreu um aumento do ponto máximo de pressão no calcanhar (Pico 1) em relação ao ponto máximo de pressão nos metatarsos (Pico 2). Em um estudo feito com 13 gestantes que foram avaliadas uma vez em cada trimestre gestacional, também foi observado que o ponto máximo de pressão na área do calcanhar aumentou em relação ao ponto máximo de pressão da área dos metatarsos no decorrer da gestação, ao contrário do que ocorre na marcha em condições normais. Os autores atribuíram a esse fato as progressivas alterações sofridas pelos pés ao longo da gestação $0^{23}$, o que pode justificar os resultados também encontrados no nosso estudo.

Para caracterizar as alterações sofridas nos pés, foi observado em um estudo com 100 gestantes que $80 \%$ relataram edema no tornozelo. Além disso, $78 \%$ referiram aumento na largura dos pés. Nossos resultados mostraram que, no decorrer da gestação, essas alterações também foram frequentes, tanto com relação ao edema quanto ao aumento da largura dos pés. Como possível causadora desses fatores está a atuação do hormônio relaxina, que leva à frouxidão ligamentar e ao acúmulo de líquido ${ }^{24}$.

A diferença entre os valores máximo e mínimo do componente horizontal da força de propulsão na direção mediolateral (máx-mín Fx) aumentou conforme os trimestres gestacionais e quando comparados com o valor de referência de $10 \%$ do peso corporal também se observa um aumento ${ }^{22}$. Isso pode significar que a gestante oscilou mais para os lados com a evolução da gestação, possivelmente na tentativa de aumentar a estabilidade postural. A estabilidade mediolateral pode estar diminuída por causa do deslocamento anterior do centro de gravidade, em consequência do ganho de massa. Esse deslocamento gera um aumento na base de apoio em até $30 \%$ com o decorrer da gestação ${ }^{14}$. Outro fator é o aumento na largura e edema nos pés, que também podem levar a uma instabilidade mediolateral. Esses resultados condizem com os encontrados por Limbery e Gilleard ${ }^{25}$, que constataram uma tendência de oscilação mediolateral, de forma que as gestantes buscam maior estabilidade na fase de apoio do CM.

Diante deste quadro, nota-se que ocorreram muitas alterações físicas na gestação. Com relação ao quesito "qualidade de vida", pode-se observar que o domínio físico é o que mais decresceu em porcentagem de respostas, principalmente no terceiro trimestre gestacional. $\mathrm{O}$ domínio físico do Questionário Whoqol-bref apresenta a percepção da gestante quanto à dor e desconforto; energia e fadiga; sono e repouso; mobilidade; atividades de vida cotidiana; dependência de medicação ou de tratamento; e capacidade de trabalho ${ }^{19}$.

Associada a essa redução na qualidade de vida, foi observado que as alterações na marcha, como a diminuição da velocidade, levam ao aparecimento de dor pélvica, por causar maior rotação da articulação sacroilíaca, ocasionando dores e desconfortos musculoesqueléticos ${ }^{26}$. McCrory et al. ${ }^{27}$ evidenciaram que durante a gestação, e principalmente no terceiro trimestre, a gestante apresenta grande instabilidade postural dinâmica. Esse quadro gera riscos de queda equivalentes ao de uma idosa com mais de 70 anos de idade, podendo acarretar lesões como fraturas, entorses, luxações, ruptura de órgãos internos, descolamento prematuro da placenta, embolia do líquido amniótico e, ocasionalmente, óbito do feto e da própria gestante $^{28}$.

Além disso, foi constatado que o caminhar é o exercício mais recomendado e o mais realizado pelas gestantes (cerca de $43 \%$ preferem o caminhar como forma de se exercitar) ${ }^{29}$. Por isso é necessário reconhecer as alterações da marcha 
ocorridas durante a gestação de forma que não causem compensações ou a aquisição de posições antálgicas, pois assim os benefícios do exercício físico serão alcançados, tanto pela gestante quanto pelo feto.

Portanto, ocorreram alterações na marcha logo no primeiro trimestre gestacional, que aumentaram ou permaneceram no decorrer da gestação. Os ganhos de massa corporal, em conjunto com a presença de edema nos pés, tornam-se fatores que interferem diretamente nessas alterações, como aumento do tempo de apoio do calcanhar e diminuição da força de propulsão. Na aplicação clínica é necessário enfatizar a prática de atividade física voltada para a melhora do equilíbrio através da manutenção do tônus e força muscular, prevenção de quedas e bem-estar físico durante a gestação, proporcionando melhora da qualidade de vida.

\section{Referências}

1. Heckman JD, Sassard R. Musculoskeletal considerations in pregnancy. J Bone Joint Surg Am. 1994;76(1 1):1720-30.

2. Ireland ML, OH SM. The effects of pregnancy on the musculoskeletal system. Clin Orthop Relat Res. 2000;(372): 169-79.

3. Nyska M, Sofer D, Porat A, Howard CB, Levi A, Meizner I. Planter foot pressures in pregnant women. Isr J Med Sci. 1997;33(2): 136-46.

4. Tsung BY, Zhang M, Fan YB, Boone DA. Quantitative comparison of plantar foot shapes under different weight-bearing conditions. J Rehabil Res Dev. 2003;40(6):517-26.

5. Alvarez R, Stokes IAF, Asprinio DE, Trevino S, Braun T. Dimensional changes of the feet in pregnancy. J Bone Joint Surg Am. $1988 ; 70(2): 271-4$.

6. Borg-Stein J, Dugan SA. Musculoskeletal disorders of pregnancy, delivery and postpartum. Phys Med Rehabil Clin N Am. 2007; 18(3):459-76.

7. Marnach ML, Ramin KD, Ramsey PS, Song SW, Stensland JJ, An KN. Characterization of the relationship between joint laxity and maternal hormones in pregnancy. Obstet Gynecol. 2003;101(2):331-5.

8. Schauberger CW, Rooney BL, Goldsmith L, Shenton D, Silva PD, Schaper A. Peripheral joint laxity increases in pregnancy but does not correlate with serum relaxin levels. Am J Obstet Gynecol. 1996; 174(2):667-71.

9. Abrams B. Weight gain and energy intake during pregnancy. Clin Obstet Gynecol. 1994;37(3):515-27.

10. To WW, Cheung $W$. The relationship between weight gain in pregnancy, birth-weight and postpartum weight retention. Aust N Z J Obstet Gynaecol. 1998;38(2):176-9.

11. Ritchie JR. Orthopedic considerations during pregnancy. Clin Obstet Gynecol. 2003;46(2):456-66.

12. Meglan D, Todd F. Kinetics of human locomotion. In: Rose J, Glambe JG, editors. Human walking. Baltimore: Williams \& Wilkins; 1994. p. 73-99.

13. Butler EE, Colón I, Druzin ML, Rose J. Postural equilibrium during pregnancy: decreased stability with an increased reliance on visual cues. Am J Obstet Gynecol. 2006; 195(4): $1104-8$.

14. Bird AR, Menz HB, Hyde CC. The effect of pregnancy on footprint parameters. A prospective investigation. J Am Podiatr Med Assoc. 1999;89(8):405-9.

15. Foti T, Davids JR, Bagley A. A biomechanical analysis of gait during pregnancy. J Bone Joint Surg Am. 2000;82(5):625-32.

16. Alexander GR, Tompkins ME, Cornely DA. Gestational age reporting and preterm delivery. Public Health Rep. 1990;105(3):267-75.
17. Rossavik IK, Fishburne Jl. Conceptional age, menstrual age, and ultrasound age: a second trimester comparison of pregnancies of known conception date with pregnancies dated from the last menstrual period. Obstet Gynecol. 1989;73(2):243-9.

18. Reis FA, Ribeiro EA, Carvalho PTC, Belchior ACG, Arakaki JC, Vasconcelos RA. Análise da confiabilidade do método Figura Oito e da volumetria para mensuração do edema de tornozelo. Rev Bras Med Esporte. 2004; 10(6):468-71.

19. Fleck MPA, Louzada S, Xavier M, Chachamovich E, Vieira G, Santos $L$, et al. Aplicação da versão em português do instrumento abreviado de avaliação da qualidade de vida "WHOQOL-bref". Rev Saúde Pública. 2000;34(2):178-83.

20. Fleck MPA, Louzada S, Xavier M, Chachamovich E, Vieira G, Santos $L$, et al. Desenvolvimento da versão em português do instrumento de avaliação de qualidade de vida da Organização Mundial da Saúde (WHOQOL-100). Rev Bras Psiquiatr. 1999;21(1):19-28.

21. Golomer E, Ducher D, Arfi GS, Sud R. Simple locomotion and during load carrying in pregnant woman. J Gynecol Obstet Biol Reprod (Paris). $1991 ; 20(3): 406-12$.

22. Perry J. Análise da marcha: marcha normal. Barueri: Manole; 2004. vol. 1.

23. Goldberg J, Besser MP, Selby-Silverstein L. Changes in foot function throughout pregnancy. Obstet Gynecol. 2001;97/4 Suppl 1):S39.

24. Ponnapula P, Boberg JS. Lower extremity changes experienced during pregnancy. J Foot Ankle Surg. 2010;49(5):452-8.

25. Lymbery JK, BAppSci(Physiother)Hons, Gilleard W. The stance phase of walking during late pregnancy: temporospatial and ground reaction force variables. J Am Podiatr Med Assoc. 2005;95(3):247-53.

26. Wu W, Meijer OG, Lamoth CJC, Uegaki K, van Dieën JH, Wiusman PI, et al. Gait coordination in pregnancy: transverse pelvic and thoracic rotations and their relative phase. Clin Biomech. 2004; 19(5):480-8.

27. McCrory JL, Chambers AJ, Daftary A, Redfern MS. Dynamic postural stability during advancing pregnancy. J Biomech. 2010;43(12):2434-9.

28 Dunning K, Lemasters $G$, Bhattacharya a. A major public health issue: the high incidence of falls during pregnancy. Matern Child Health J. 2010;14(5):720-5.

29. Clapp JF 3rd. Exercise during pregnancy: a clinical update. Clin Sports Med. 2000;19(2):273-86. 